Eq. (15) is attained at $t=0.01,0.018$, and $0.024 \mu \mathrm{m}$ for $\sigma=50,75$, and $100(\mathrm{eV})^{-1}$, respectively. These results are illustrated in Fig. 3, where we have graphed $\left(\xi_{m}\right)_{t h}$ vs $t$ for this laser. Similarly if $\sigma=100(\mathrm{eV})^{-1}, \Gamma=0.3$ (for $t \approx 0.1$ $\mu \mathrm{m}), \alpha_{l}=10 \mathrm{~cm}^{-1}, R_{1}=R_{2}=0.32$, then $L$ must be greater than $38 \mu \mathrm{m}$.

Several other restrictions apply in comparing Eqs. (11)(15) with experimental observations. First, if the waveguide is not exactly symmetrical, the mode becomes cutoff before $t$ attains zero, that is $\Gamma \rightarrow 0$ for $t>0$, such that $I_{\mathrm{th}} \rightarrow \infty$ at some small positive value of $t$. All the equations, but (13), are valid for the nonsymmetric waveguide. In addition for very thin active regions $\eta_{i}$ may not be constant and lastly quantum well effects ${ }^{13}$ become important for active region thicknesses below about $t=0.04 \mu \mathrm{m}$. Under those circumstances the exponential form of the absorption edge, from which our results were developed, is no longer an adequate description.

In summary, by using a simple expression for the bandto-band absorption of the active region material in a diode laser we have formulated an equation for the modal gain and employed that result to derive simple formulas for threshold current density and lasing wavelength as functions of the device parameters, including active region thickness, laser length, etc.

${ }^{1}$ G. Lucovsky, A. J. Varga, and R. F. Schwarz, Solid State Commun. 3, 9 (1965).

${ }^{2}$ C. J. Hwang, J. Appl. Phys. 40, 3731 (1969).

${ }^{3}$ H. C. Casey, Jr. and M. B. Panish, Heterostructure Lasers (Academic, New York, 1978).

${ }^{4}$ C. H. Henry, R. A. Logan, and F. R. Merrit, J. Appl. Phys. 51, 3042 (1980).

${ }^{5}$ K. Petermann, IEEE J. Quantum Electron. QE-15, 566 (1979).

${ }^{6}$ W. Streifer, D. R. Scifres, and R. D. Burnham, Jpn. J. Appl. Phys. 21, L $282(1982)$

${ }^{7}$ H. C. Casey, Jr. and F. Stern, J. Appl. Phys. 47, 631 (1976).

${ }^{8}$ B. W. Hakki and T. L. Paoli, J. Appl. Phys. 46, 1299 (1975).

${ }^{9}$ E. Pinkus, B. I. Miller, I. Hayashi, and P. W. Foy, J. Appl. Phys. 43, 2827 (1972).

${ }^{10} \mathrm{G}$. Lucovsky, Appl. Phys. Lett. 5, 37 (1964).

${ }^{11}$ W. T. Tsang, Appl. Phys. Lett. 36, 11 (1980).

${ }^{12}$ D. R. Scifres, R. D. Burnham, and W. Streifer (unpublished).

${ }^{13}$ R. Dingle, W. Wiegmann, and C. H. Henry, Phys. Rev. Lett. 33, 827 (1974).

\title{
InGaAsP/InP undercut mesa laser with planar polyimide passivation
}

\author{
U. Koren, T. R. Chen, C. Harder, A. Hasson, K. L. Yu, L. C. Chiu, S. Margalit, and \\ A. Yariv \\ California Institute of Technology, Pasadena, California 91125
}

(Received 1 November 1982; accepted for publication 23 November 1982)

An undercut mesa laser is fabricated on an $n^{+}$-InP substrate using a single step liquid phase epitaxy growth process and a planar structure is obtained by using a polyimide filling layer. The lasers operate at fundamental transverse mode due to a scattering loss mechanism. Threshold currents of $18 \mathrm{~mA}$ and stable single transverse mode operating at high currents are obtained.

PACS numbers: 42.55.Px

Recently, a novel mass transport technique has been utilized for fabrication of low threshold InGaAsP/InP laser diodes. ${ }^{1,2}$ This technique, which was first reported in Ref. 1, consists of selectively etching an undercut mesa structure and then creating a buried waveguide by filling the undercut region with mass transported InP. It has also been reported ${ }^{3}$ that a terrace mesa structure with similar undercut, but without the mass transport process also yielded low threshold lasers. Here we report an undercut mesa structure on an $n^{+}$-InP substrate where the undercut regions are filled with $\mathrm{SiO}_{2}$ and polyimide ${ }^{4}$ polymer layers. The use of polyimide in optoelectronic circuits was first suggested ${ }^{5}$ for the fabrication of interconnection waveguides on top of InP wafers. This work demonstrated the potential importance of this material for integrated optoelectronic circuits. Here, we incorporated the polyimide layer in the laser structure to form a very strongly index-guided laser. In such a waveguide, the transverse modal behavior is controlled by the scattering loss mechanism. This has been reported by several authors ${ }^{6,7}$ for $\mathrm{GaAs}$ lasers and this is also observed in our results.
The advantages of using the polyimide material are that it can withstand the high-temperature treatment required for contact annealing and that it forms planar layers with excellent adhesion, which are also free of pin holes and cracks and are mechanically tough and environmentally resistant. Also this layer can be photolithographically processed before final curing. Some grades of the polyimide material (e.g., Pyralin 2555) have considerable amounts of optical absorption. It is of the order of $20-40 \mathrm{~cm}^{-1}$ for $1.3-$ $1.2-\mu \mathrm{m}$ wavelengths, respectively (depending also on curing conditions).

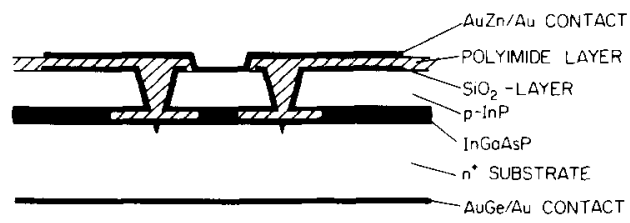

FIG. 1. Schematic structure of an undercut mesa laser. 


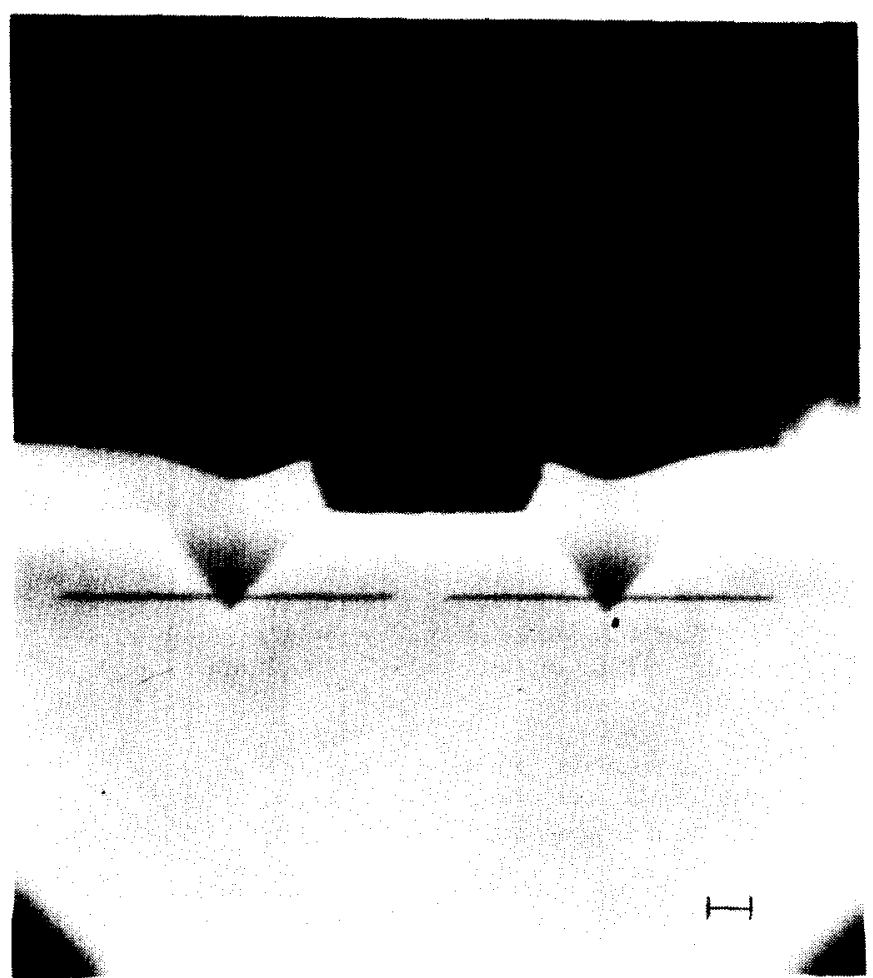

FIG. 2. SEM photomicrograph of the laser cross section. Scale is $1 \mu \mathrm{m}$.

It has been experimentally observed that when the undercut region near the InGaAsP active layers is filled with this grade of polyimide, the lasers exhibited hysteresis in the light-current characteristics and showed bistable operation. The absorption at the sides of the waveguide can be practically eliminated by using a $\mathrm{SiO}_{2}$ intermediate layer, or by using optical grade polyimide (Pyralin 2566) which has much lower optical absorption at this spectral range.

The schematic structure of the undercut mesa laser is shown in Fig. 1. It is made with a single step liquid phase epitaxial (LPE) growth process. Three layers are grown on an $n^{+}$-InP substrate: $n^{+}-\operatorname{InP}$ (tin doped to $2 \times 10^{18} \mathrm{~cm}^{-3}$ ), InGaAsP (undoped), and P-InP (Zn doped to $2 \times 10^{17}$ $\mathrm{cm}^{-3}$ ). After growth, a shallow $\mathrm{Zn}$ diffusion is performed. Two $\mathrm{V}$ grooves are etched using Br-methanol etching through $\mathrm{Si}_{3} \mathrm{~N}_{4}$ stripe openings in the [011] direction. The InGaAsP layer between the two $\mathrm{V}$ grooves is then selectively

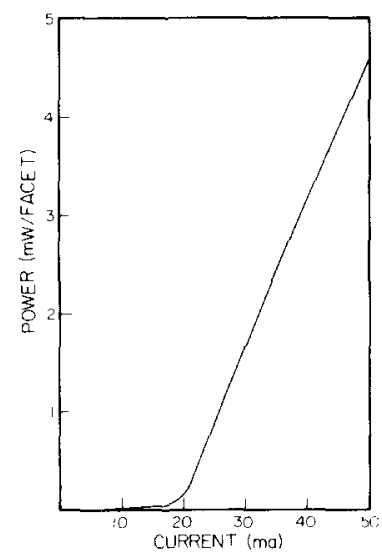

FIG. 3. Light vs current characteristics for an undercut mesa laser with a 2000 $\AA$-thick intermediate $\mathrm{SiO}_{2}$ layer.

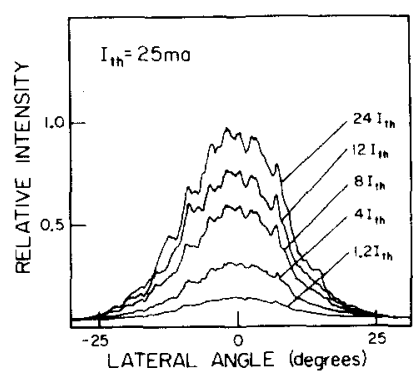

FIG. 4. Far-field patterns for the undercut mesa laser.

undercut using the staining solution $\mathrm{KOH}$ : $\mathrm{K}_{3} \mathrm{Fe}(\mathrm{CN})_{6}: \mathrm{H}_{2} \mathrm{O}, 1: 2: 6$.

The undercut etching is stopped when the quaternary layer is about $1-1.5 \mu \mathrm{m}$ wide. Then, $\mathrm{a} \mathrm{SiO}_{2}$ layer is deposited by chemical vapor deposition (CVD) and the polyimide layer is deposited by spinning. These layers are photolithographically removed from the $p$-contact area (see Fig. 1). Following curing the polyimide layer at $400^{\circ} \mathrm{C}$, the $p$ and $n$ contacts are made by conventional techniques. A scanning electron microscopic (SEM) photomicrograph of the laser cross section is shown in Fig. 2. After thinning, the wafer is cleaved but the cleaved crystals are still held together by the polyimide film. The small laser wafers are then separated by cutting the polyimide film using a blade that is inserted from the substrate side.

Light versus current characteristics are shown in Fig. 3. These lasers have threshold currents in the range of 20-30 $\mathrm{mA}$ for $250-\mu \mathrm{m}$ cavity length and the lowest threshold current observed was $18 \mathrm{~mA}$ for this cavity length. The threshold currents are somewhat higher than those reported for mass transport lasers ${ }^{1,2}$ and for the terrace mesa laser. ${ }^{3}$ The reason may be due to the considerable scattering loss from the sidewalls of the symmetric waveguide which is enhanced by the large difference in the index of refraction between the active layer and the cladding $\mathrm{SiO}_{2}$ layer $(n \simeq 1.5)$. The scattering from the sidewalls creates a distinct pattern in the farfield distributions as shown in Fig. 4. The scattering pattern does not change as optical power is increased. Fundamental mode operation prevails because scattering losses are much lower than for higher order modes. ${ }^{6}$ Figure 4 shows stable fundamental mode operation up to $I=24 I_{\text {th }}$. A typical temperature dependence of the threshold current is shown in Fig. 5, showing $T_{0}=78^{\circ} \mathrm{K}$ at room temperature. The $T_{0}$ values are somewhat higher than for buried heterostructure lasers because leakage current is eliminated in the present mesa laser.

When absorbing grade of polyimide (Pyralin 2555) was used for filling the undercut of the waveguide (without an intermediate $\mathrm{SiO}_{2}$ layer), lasers with hysteresis in the light-

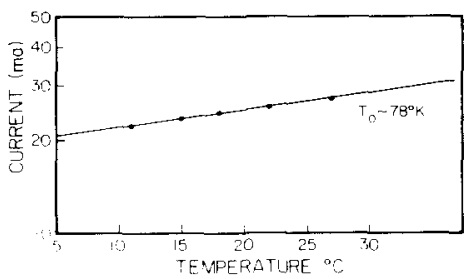

FIG. 5. Temperature dependence of the threshold current for an undercut mesa laser. 

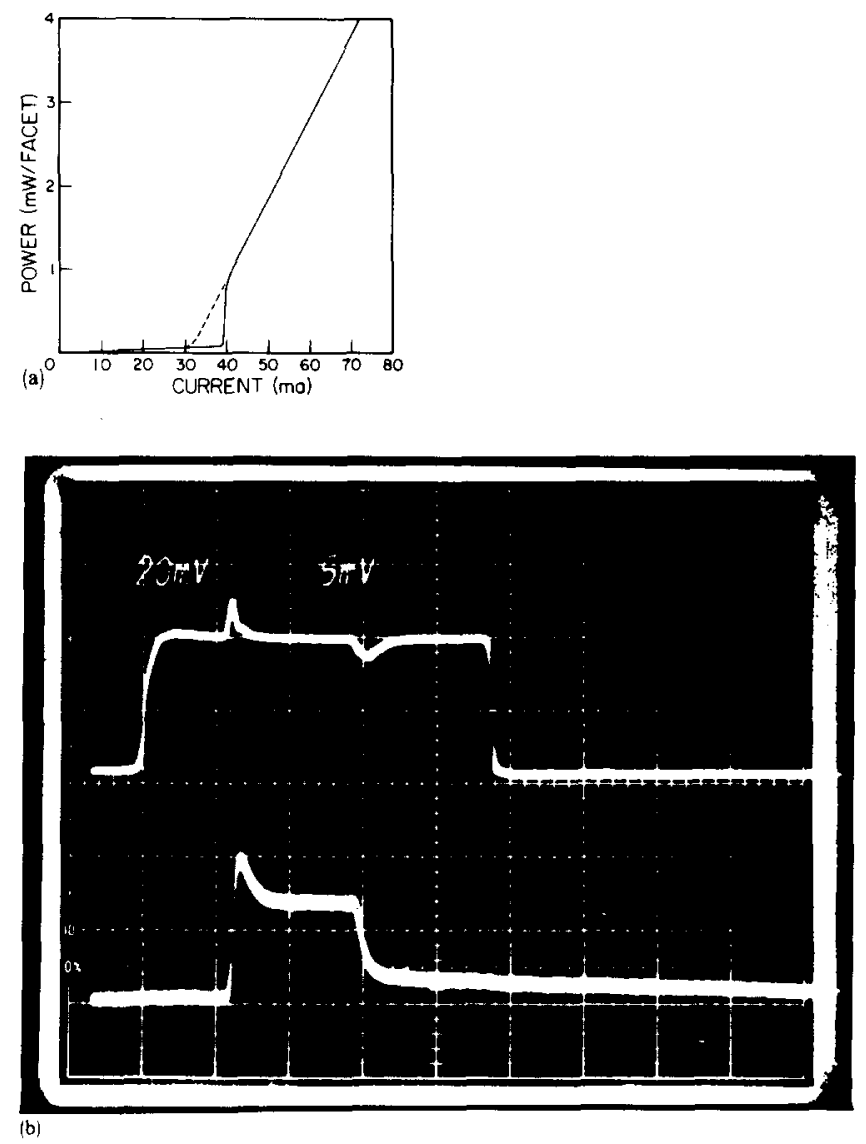

FIG. 6. (a) Light vs current characteristics for a laser with a Pyralin 2555 polyimide layer and no intermediate $\mathrm{SiO}_{2}$ layer. (b) Light response (lower trace) for a current pulse (upper trace) with superimposed on-off trigger pulses. Horizontal scale: - $100 \mathrm{~ns} /$ div; current scale: $20 \mathrm{~mA} / \mathrm{div}$. current characteristics were obtained as shown in Fig. 6(a). These lasers are bistable for currents within the hysteresis loop. Bistable operation is shown in Fig. 6(b) where the light pulse is triggered on and off by small pulses that are superimposed on the current pulse. The noise spectrum of these lasers was measured using a fast InGaAs $P I N$ diode. No pulsation could be observed at a frequency range of dc $2 \mathrm{GHz}$. Measurement of the optical spectrum showed single longitudinal mode operation up to $1.6 I_{\text {th }}$. Further details and discussion of the bistable lasers will be presented in a later publication.

In conclusion, undercut mesa lasers have been studied. Fabrication of the lasers is simple and requires only one LPE growth step similar to the first step in buried heterostructure laser fabrication. A planar structure is obtained by the use of a filling polyimide layer. Stable fundamental transverse mode operation and relatively high $T_{0}$ are obtained for these lasers.

This work was supported by the Office of Naval Research and by the Air Force Office of Scientific Research.

${ }^{1}$ Z. L. Liau and J. N. Walpole, Appl. Phys. Lett. 40, 568 (1982).

${ }^{2}$ T. R. Chen, L. C. Chiu, K. L. Yu, U. Koren, A. Hasson, S. Margalit, and A. Yariv, Appl. Phys. Lett. 41, 1115(1982).

${ }^{3}$ T. R. Chen, L. C. Chiu, K. L. Yu, U. Koren, A. Hasson, S. Margalit, and A. Yariv, IEEE J. Quantum Electron. May 1983.

${ }^{4}$ Pyralin, Polyimide Coatings for Electronics, DuPont Company, Wilmington, DE 19898.

${ }^{5}$ K. Furuya, B. I. Miller, L. A. Coldren, and R. E. Howard, “A Novel Deposit/Spin Waveguide Interconnection (DSWI) for Semiconductor Integrated Optics Design and Material," Integrated and Guided Wave Optics Conference January 1982.

${ }^{6}$ C. H. Henry, R. A. Logan, and F. R. Merritt, J. Quantum Electron. QE17, 2196 (1981).

${ }^{7}$ D. Kerps, J. Quantum Electron. QE-18, 1113 (1982). 